\title{
Clinical Response with Sunitinib Therapy in the Treatment of Anaplastic Thyroid Cancer
}

\author{
Kenneth M. Wong ${ }^{1}$, Theodore Scott Nowicki ${ }^{2}$, Carmelo Puccio ${ }^{3}$, Tauseef Ahmed ${ }^{3}$, Raj Tiwari ${ }^{2}$, \\ Jan Geliebter ${ }^{2}$, Augustine Moscatello ${ }^{4}$ \\ ${ }^{1}$ Department of Otolaryngology—Head and Neck Surgery, New York Eye and Ear Infirmary, New York, USA; ${ }^{2}$ Department of \\ Microbiology and Immunology, New York Medical College, Valhalla, USA; ${ }^{3}$ Department of Medical Oncology, Westchester \\ Medical Center, Valhalla, USA; ${ }^{4}$ Department of Otolaryngology_-Head and Neck Surgery, Westchester Medical Center, Valhalla, \\ USA. \\ Email: kwong@nyee.edu
}

Received February 28 ${ }^{\text {th }}, 2012$; revised March $31^{\text {st }}, 2012$; accepted April $15^{\text {th }}, 2012$

\begin{abstract}
Background: Anaplastic thyroid cancer (ATC), while rare, carries a uniformly poor prognosis. Current treatment includes surgery when possible, radiotherapy, and chemotherapy. Multiple chemotherapeutic agents are in the process of clinical testing, and promising agents include those in the tyrosine kinase inhibitor family. Our patient represents a novel case of ATC treated with sunitinib, one such tyrosine kinase inhibitor. Methods/Results: We utilized the experimental sunitinib in conjunction with radiation therapy to treat a patient with aggressive ATC in whom curative resection was unable to be achieved due to carotid sheath and tracheal involvement. The patient had marked clinical response and sustained stable disease for 8 months, which coincides with reported data regarding sunitinib to treat other thyroid malignancies. Conclusion: Our case illustrates the efficacy of sunitinib therapy as a possible adjunct in the treatment of ATC.
\end{abstract}

Keywords: Sunitinib; Anaplastic Thyroid Cancer; Tyrosine Kinase Inhibitor; Chemotherapy; ATC

\section{Introduction}

Anaplastic thyroid cancer (ATC) is the least common thyroid malignancy, but carries an extremely poor prognosis. The female to male ratio is $5: 1$ and peak incidence is in the sixth and seventh decade of life [1]. Median life expectancy after diagnosis is only 3 - 9 months, with two year survival rates at only $10 \%-15 \%$ [2]. Presenting symptoms are usually a rapidly enlarging neck mass with associated symptoms of dyspnea, hoarseness, or dysphagia. ATC has a propensity for rapid progression; approximately $20 \%-50 \%$ of patients have distant metastasis at the time of diagnosis, and 25\% develop new metastasis during the course of the disease [3]. Although not standardized, management of ATC ideally involves multimodality treatment (consisting of surgery, radiotherapy, and chemotherapy) to maximize clinical outcome, as such management paradigms have been shown to improve survival times [4]. Additionally, multiple studies have shown that performing curative resection confers increased one-year survival rates, as well as increased overall survival times [3,5].

Multiple chemotherapy agents have been used in the treatment of ATC, and many more are in the process of clinical testing. One such experimental agent is sunitinib (SU011248, Sutent), which is a tyrosine kinase inhibitor with activities against vascular endothelial growth factor receptor (VEGFR) types 1 and 2, platelet-derived growth factor receptor (PDGFR) alpha and beta, c-KIT, and RET. It is FDA-approved for use against renal cell carcinoma and imatinib-refractory gastrointestinal stromal tumors, but its use has been broadened as the molecular biology of thyroid cancer becomes better understood [6]. Several phase II trials involving sunitinib are being conducted on patients with medullary thyroid cancer (MTC) and well differentiated thyroid cancer (WDTC). Preliminary results published thus far have been promising: sunitinib treatment has been shown to increase partial response rates and duration of stable disease in MTC and WDTC patients alike [7-9]. There have also been multiple individual case reports of WDTC patients with prolonged disease stabilization after treatment with sunitinib, even in the face of distant metastasis [10-12].

However, there are no published reports dealing with the use of sunitinib to improve the clinical course of ATC. Here we report on a patient with aggressive ATC who was treated with sunitinib, which resulted in a marked 
clinical response and prolonged stable disease.

\section{Case Report}

The patient is a 54-year-old female who was transferred to Westchester Medical Center in May 2011 with increasing respiratory distress, stridor, shortness of breath, and a rapidly enlarging neck mass during a six week period. She complained of difficulty sleeping in the left lateral decubitus position and had dysphagia for solids. Past medical history was significant for Lyme disease and anxiety. The patient is a current smoker with 15 pack/year history and denied alcohol and intravenous drug use. She denied exposure of external radiation to the neck or radiotherapy treatment in the past. Physical exam revealed a large approximately $6 \times 6 \mathrm{~cm}$ firm mass in the central neck with extensive bilateral cervical lymphadenopathy greater on the right than the left. Computed tomography (CT) scan of the neck revealed a large lesion arising from the thyroid bed, with tracheal deviation to the left, extensive lymphadenopathy (right greater than left) with compression of right internal jugular vein, and large mass abutting the right carotid artery (Figure 1). CT of the chest/abdomen/pelvis did not reveal any evidence of metastatic disease. Fine needle aspiration performed from the referral hospital was inconclusive, but suggestive of medullary thyroid cancer.

The patient was taken to the operating room, where a tracheotomy with subtotal thyroidectomy was performed. Intraoperatively, there was gross disease involving the right carotid sheath and approximately $3 \mathrm{~cm}$ of tracheal wall invasion. Final pathological diagnosis was poorly differentiated thyroid carcinoma, 25\% anaplastic component, with spindle and giant cells, and a minor component of papillary columnar cell carcinoma. Figure 2 shows post-surgical changes prior to radiotherapy and chemotherapy. The patient underwent radiation therapy and received a total dose of 5400 cGy to the thyroid bed and bilateral necks. She also received one cycle of sunitinib malate $50 \mathrm{mg}$ daily for four weeks on/two weeks off beginning May 2011. Complications related to sunitinib therapy experienced by our patient included hand-foot syndrome. She developed a rash on her wrists and plantar surface of her foot, which was treated with Bacitracin and triamcinolone cream. The nadir for her white blood cell count and platelets during treatment was $2.6 \times 10^{3} / \mathrm{mm}^{3}$ and $90 \times 10^{3} / \mathrm{mm}^{3}$, respectively.

Clinical response was dramatic after the first cycle of sunitinib therapy. The bilateral cervical lymphadenopathy decreased clinically and became non-palpable. Repeat CT scan of the neck after the first cycle showed marked decrease in size of cervical lymphadenopathy (Figure 3). She received subsequent cycles of chemotherapy at $37.5 \mathrm{mg}$ bid and has had stable disease on that

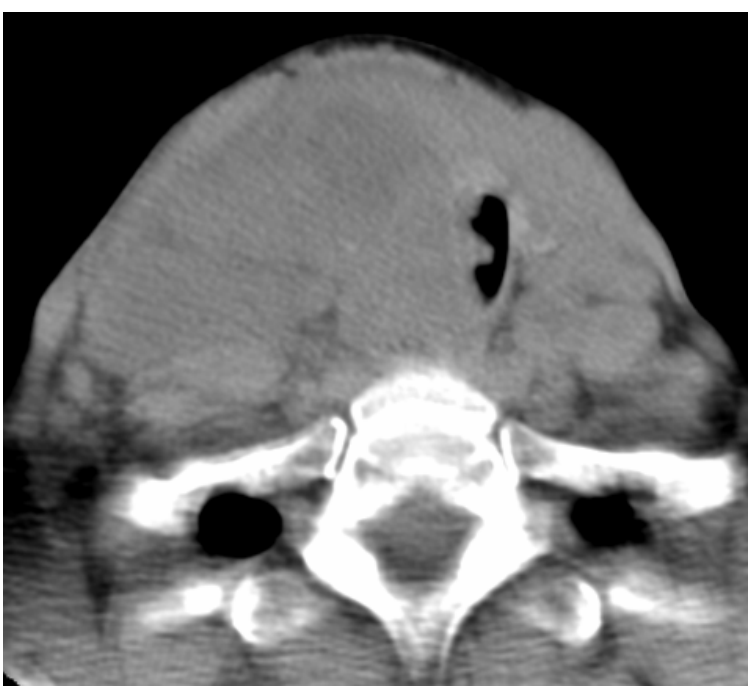

Figure 1. Pre-treatment CT scan.

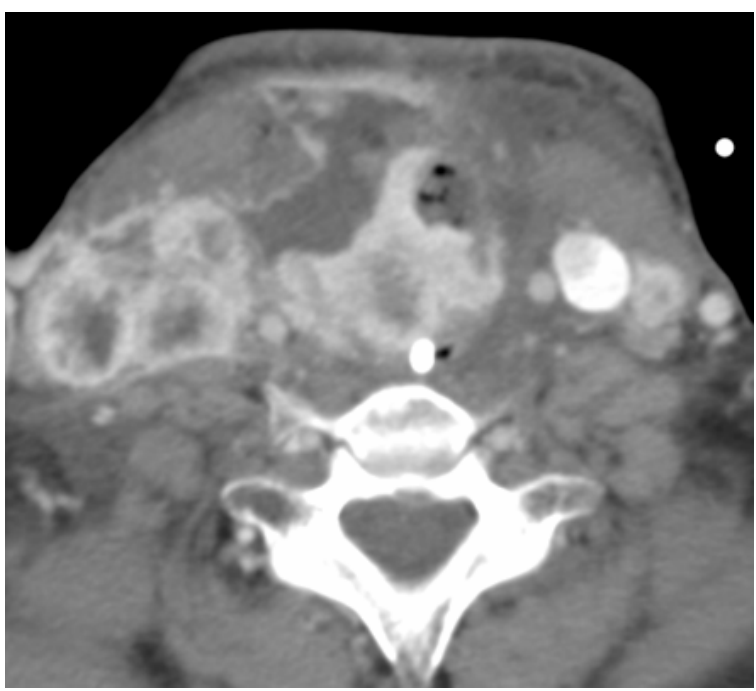

Figure 2. Post-op CT scan before radiation or chemotherapy treatment.

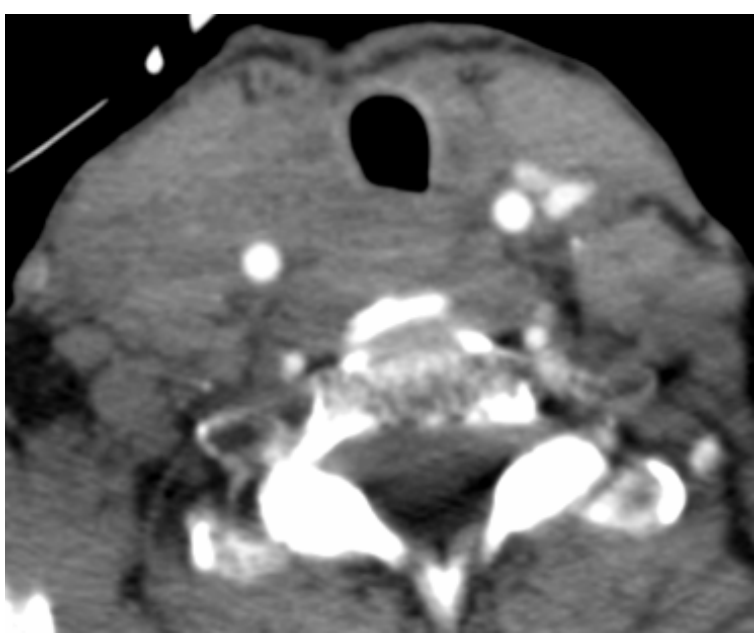

Figure 3. CT scan after one cycle of sunitinib therapy. 
chemotherapy regimen. However, she was readmitted December 2011 with tracheotomy complications and was found to have a trachea-esophageal fistula at the distal tip of the tracheotomy tube. On repeat CT scan, recurrence of lymphadenopathy was seen in the right cervical neck (Figure 4). She also had a left pleural effusion which was drained, and there was no evidence of malignant cells in the pleural fluid.

\section{Discussion}

Given the dismal prognosis of ATC and the limited ability of surgeons to perform curative resection, specific inhibitors of molecular mediators of ATC pathogenesis have received an increasing amount of attention. This approach has been facilitated in large part by ongoing research into the molecular alterations which commonly contribute to ATC progression [13]. One widely held view is that ATC arises from WDTC subtypes, namely papillary thyroid carcinoma (PTC) and follicular thyroid carcinoma (FTC). Following the initial mutagenic events in PTC and FTC, loss-of-function p53 mutations, as well as numerous other gross chromosomal abnormalities, are thought to be the driving force behind the emergence of the aggressive and rapid progressing phenotype of ATC [14-16].

The mutations which ultimately drive the progression from WDTC to ATC under this model can occur in a widely variant manner (with differences being documented among large subsets of patients) [17]. Therefore, one novel approach to therapy is to target the molecular factors present in the supposed precursor WDTC lesions

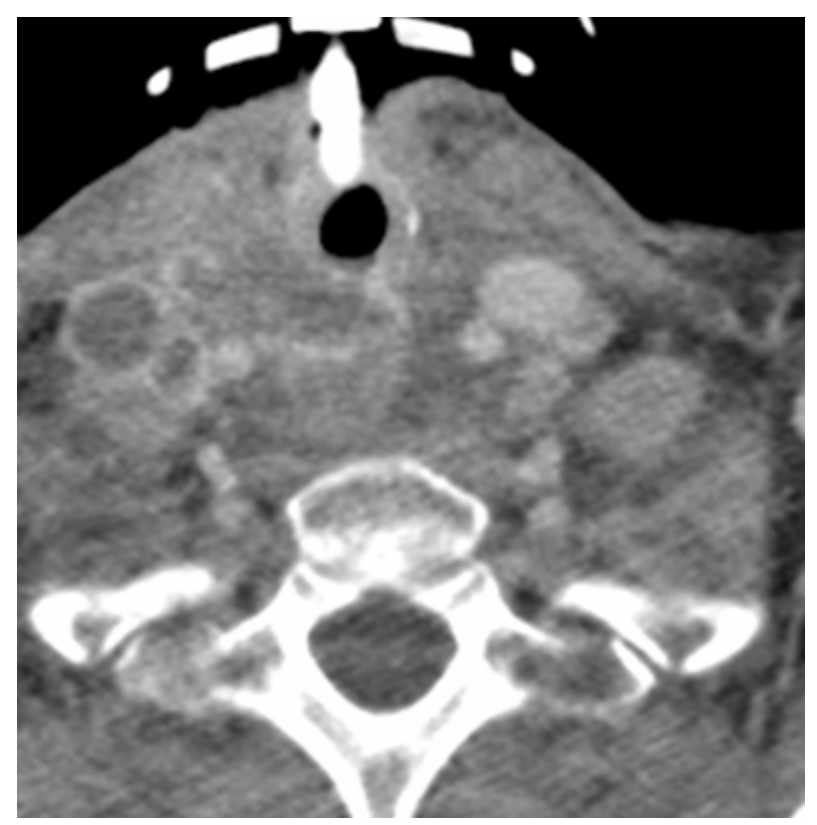

Figure 4. CT scan at readmission with right cervical lymphadenopathy. which remain active in ATC. Several recent studies have demonstrated that the PI3K/Akt signaling pathway, which is critically important to growth and invasion in WDTC (particularly PTC), remains activated in ATC as well $[18,19]$. By targeting this and other pathways with specific molecular inhibitors, such as sunitinib, it may be possible to reduce these growth and invasion behaviors in ATC as well. Indeed, recent studies have demonstrated that sunitinib is able to target the PI3K/Akt signaling pathway in other malignancies, resulting in impressive reductions in cellular growth $[20,21]$.

Our patient demonstrated a marked improvement following treatment with sunitinib. Even though curative resection of the tumor was unable to be achieved due to involvement of the carotid sheath and trachea, her prolonged state of stable disease for 8 months is comparable to the reported increased median survival in patients receiving triple modality therapy compared to chemotherapy alone or radiotherapy alone (11 months vs. 5.7 months vs. 4 months, respectively) [4]. Another important study by Haigh et al. found median survival of 43 months versus 3 months $(p=0.002)$ in patients receiving curative resection plus adjuvant chemoradiotherapy compared to those who underwent palliative resection and adjuvant chemoradiotherapy, respectively [5]. The addition of sunitinib in our patient likely contributed to the favorable clinical outcome of controlling disease progression or recurrence.

It is worth pointing out that the patient's positive response to sunitinib therapy goes against a recent report by D'Agostino et al. in which sunitinib failed to exert a significant effect on ATC cell growth and proliferation in vitro [22]. However, it is possible that in vitro studies are unable to completely approximate a given ATC tumor's behavior as it would exist within the tumor microenvironment in vivo. Indeed, another recent report by Gule et al. demonstrated that direct inhibition of VEGFR-2, a molecular target of sunitinib, was able to significantly reduce the growth of ATC cells in orthotopic mouse xenografts [23]. This paper also utilized a large number ATC cell lines which were different from those studied by D'Agostino et al., highlighting the degree of genetic diversity which can be seen between individual tumors in ATC, as well as the difficulty faced in generalizing and extrapolating data derived from cellular models of this malignancy.

\section{Conclusion}

While the multi-targeted tyrosine kinase inhibitor sunitinib has been shown to be efficacious in the treatment of WDTC and MTC, its role in the treatment of ATC needs to be further studied. Our patient represents the first reported case utilizing sunitinib in the treatment course of 
ATC. The period of 8 months with stable disease is comparable to reports in the current literature of patients undergoing similar multimodality treatment, especially considering that curative resection was not achievable. Our case illustrates the efficacy of sunitinib as an adjunct to the treatment of ATC. Further clinical trials are needed to validate and define the clinical benefit.

\section{REFERENCES}

[1] A. R. Shaha, "Implications of Prognostic Factors and Risk Groups in the Management of Differentiated Thyroid Cancer,” Laryngoscope, Vol. 114, No. 3, 2004, pp. 393402. doi:10.1097/00005537-200403000-00001

[2] J. Rosai, M. L. Cargangiu and R. Delellis, "Tumours of the Thyroid Gland,” In: A. P. Stout, Ed., Atlas of Tumour Pathology, 3rd Series, Fascicle 5, Armed Forces Institute of Pathology, Washington DC, 1992.

[3] J. P. Pierie, A. Muzikansky, R. D. Gaz, W. C. Faquin and M. J. Ott, "The Effect of Surgery and Radiotherapy on Outcome of Anaplastic Thyroid Carcinoma," Annals of Surgical Oncology, Vol. 9, No. 1, 2002, pp. 57-64. doi:10.1245/aso.2002.9.1.57

[4] B. Busnardo, O. Daniele, M. R. Pelizzo, et al., "A Multimodality Therapeutic Approach in Anaplastic Thyroid Carcinoma: Study on 39 Patients," Journal of Endocrinological Investigation, Vol. 23, No. 11, 2000, pp. 755761.

[5] P. I. Haigh, P. H. Ituarte, H. S. Wu, et al., "Completely Resected Anaplastic Thyroid Carcinoma Combined with Adjuvant Chemotherapy and Irradiation Is Associated with Prolonged Survival," Cancer, Vol. 91, No. 12, 2001, pp. 2335-2342.

doi:10.1002/1097-0142(20010615)91:12<2335::AID-CN CR1266>3.0.CO;2-1

[6] L. Q. Chow and S. G. Eckhardt, "Sunitinib: From Rational Design to Clinical Efficacy,” Journal of Clinical Oncology, Vol. 25, No. 7, 2007, pp. 884-896. doi:10.1200/JCO.2006.06.3602

[7] A. Ravaud, C. de la Fouchardière, J. Asselineau, et al., "Efficacy of Sunitinib in Advanced Medullary Thyroid Carcinoma: Intermediate Results of Phase II THYSU," Oncologist, Vol. 15, No. 2, 2010, pp. 212-213. doi:10.1634/theoncologist.2009-0303

[8] L. L. Carr, D. A. Mankoff, B. H. Goulart, et al., "Phase II Study of Daily Sunitinib in FDG-PET-Positive, IodineRefractory Differentiated Thyroid Cancer and Metastatic Medullary Carcinoma of the Thyroid with Functional Imaging Correlation,” Clinical Cancer Research, Vol. 16, No. 21, 2010, pp. 5260-5268. doi:10.1158/1078-0432.CCR-10-0994

[9] E. W. Cohen, B. M. Needles, K. J. Cullen, et al., "Phase 2 Study of Sunitinib in Refractory Thyroid Cancer," Journal of Clinical Oncology, Vol. 26, 2008, p. 6025.

[10] S. J. Dawson, N. M. Conus, G. C. Toner, et al., "Sustained Clinical Responses to Tyrosine Kinase Inhibitor Sunitinib in Thyroid Carcinoma,” Anticancer Drugs, Vol. 19, No. 5, 2008, pp. 547-552. doi:10.1097/CAD.0b013e3282fc6cf7
[11] J. M. Cleary, P. M. Sadow, G. W. Randolph, et al., "Neoadjuvant Treatment of Unresectable Medullary Thyroid Cancer with Sunitinib,” Journal of Clinical Oncology, Vol. 28, No. 23, 2010, pp. e390-e392. doi:10.1200/JCO.2009.27.4225

[12] P. Kaldrymides, I. Kostoglou-Athanassiou, A. Gkountouvas, E. Veniou and N. Ziras, "Partial Remission of Metastatic Papillary Thyroid Carcinoma with Sunitinib. Report of a Case and Review of the Literature,” Endocrine, Vol. 37, No. 1, 2010, pp. 6-10. doi:10.1007/s12020-009-9290-z

[13] S. M. Wiseman, H. Masoudi, P. Niblock, et al., “Anaplastic Thyroid Carcinoma: Expression Profile of Targets for Therapy Offers New Insights for Disease Treatment," Annals of Surgical Oncology, Vol. 14, No. 2, 2007, pp. 719-729. doi:10.1245/s10434-006-9178-6

[14] Y. E. Nikiforov, "Genetic Alterations Involved in the Transition from Well-Differentiated to Poorly Differentiated and Anaplastic Thyroid Carcinomas," Endocrine Pathology, Vol. 15, No. 4, 2004, pp. 319-327. doi:10.1385/EP:15:4:319

[15] R. A. DeLellis, "Pathology and Genetics of Thyroid Carcinoma,” Journal of Surgical Oncology, Vol. 94, No. 8, 2006, pp. 662-669. doi:10.1002/jso.20700

[16] R. M. Quiros, H. G. Ding, P. Gattuso, R. A. Prinz and X. $\mathrm{Xu}$, "Evidence That One Subset of Anaplastic Thyroid Carcinomas Are Derived from Papillary Carcinomas Due to BRAF and p53 Mutations,” Cancer, Vol. 103, No. 11, 2005, pp. 2261-2268. doi:10.1002/cncr.21073

[17] R. C. Smallridge, L. A. Marlow and J. A. Copland, "Anaplastic Thyroid Cancer: Molecular Pathogenesis and Emerging Therapies,” Endocrine-Related Cancer, Vol. 16, No. 1, 2009, pp. 17-44. doi:10.1677/ERC-08-0154

[18] L. Santarpia, A. K. El-Naggar, G. J. Cote, J. N. Myers and S. I. Sherman, "Phosphatidylinositol 3-kinase/akt and Ras/Raf-Mitogen-Activated Protein Kinase Pathway Mutations in Anaplastic Thyroid Cancer,” Journal of Clinical Endocrinology \& Metabolism, Vol. 93, No. 1, 2008, pp. 278-284. doi:10.1210/jc.2007-1076

[19] G. García-Rostán, A. M. Costa, I. Pereira-Castro, et al., "Mutation of the PIK3CA Gene in Anaplastic Thyroid Cancer,” Cancer Research, Vol. 65, No. 22, 2005, pp. 10199-10207. doi:10.1158/0008-5472.CAN-04-4259

[20] A. Yeramian, A. Sorolla, A. Velasco, et al., "Inhibition of Activated Receptor Tyrosine Kinases by Sunitinib Induces Growth Arrest and Sensitizes Melanoma Cells to Bortezomib by Blocking Akt Pathway,” International Journal of Cancer, Vol. 130, No. 4, 2012, pp. 967-978. doi:10.1002/ijc.26096

[21] F. Yang, V. Jove, H. Xin, M. Hedvat, T. E. Van Meter and $\mathrm{H}$. Yu, "Sunitinib Induces Apoptosis and Growth Arrest of Medulloblastoma Tumor Cells by Inhibiting STAT3 and AKT Signaling Pathways,” Molecular Cancer Research, Vol. 8, No. 1, 2010, pp. 35-45. doi:10.1158/1541-7786.MCR-09-0220

[22] M. D’Agostino, P. Voce, M. Celano, et al., "Sunitinib Exerts Only Limited Effects on the Proliferation and Differentiation of Anaplastic Thyroid Cancer Cells,” Thyroid, Vol. 22, No. 2, 2012, pp. 138-144. 
[23] M. K. Gule, Y. Chen, D. Sano, et al., "Targeted Therapy of VEGFR2 and EGFR Significantly Inhibits Growth of Anaplastic Thyroid Cancer in an Orthotopic Murine
Model," Clinical Cancer Research, Vol. 17, No. 8, 2011 pp. 2281-2291. doi:10.1158/1078-0432.CCR-10-2762 